need for a change of drug regimen. After three months' treatment only about $15 \%$ of patients will still have positive smears. Among these will be most of the patients in whom treatment is failing. If sensitivity tests on the pretreatment cultures were done only for patients still positive on microscopy at three months, when the cultures should still be available, there would be an $85 \%$ saving of laboratory work. Positive results on microscopy at six months almost certainly indicate failures of treatment, and no sensitivity tests are required for continuing treatment with one of the secondary regimens.

In individual patients the main use for sensitivity tests is for those who have previously had chemotherapy and still have a positive sputum. A detailed history of previous chemotherapy can be helpful in assessing the probability of drugresistance. But this may lead to overestimating it and unnecessarily prescribing secondary regimens, with their attendant toxic risks and higher cost. So sensitivity tests in such cases-which should be few-are valuable and can lead to economies. If the history strongly suggests that drug-resistance to one or more of the primary drugs has appeared, a secondary regimen can be given until the results of sensitivity tests are available. Unfortunately this will not be for several weeks. A quicker but equally reliable test is needed.

In developing countries the first priority for using sensitivity tests is epidemiological. With too many patients for too few clinics treatment must be simplified and standardized. To use sensitivity tests to guide treatment in individual cases is wasteful and mostly unnecessary. Very good results can be obtained without them. There are three essentials: adequate attention to the patients' history, regimens of proved efficacy, and a system of control that ensures maximum co-operation from the patients. In Brazil, for instance, 75 to $85 \%$ of "bacteriologically favourable" results were obtained among patients who reported no previous chemotherapy or treatment for less than a month. ${ }^{5}$ The patterns of sensitivity could be estimated from earlier surveys-about $90 \%$ of such patients could be expected to have cultures sensitive to two or three of the first-line drugs. With a lower proportion the results would have been worse. Thus it is essential for rational planning of standardized regimens to know the prevalence of resistance in the community. It is for this that laboratory facilities for carrying out these difficult and expensive tests should be used.

\section{Remissions in Diabetes Mellitus}

Improvement in the diabetic state by the use of dietary restriction was studied systematically in the nineteenth century by Bouchardat, Cantoni, and Naunyn, each of whom reported cases in which the patient regained carbohydrate tolerance to the point where he could remain free of glycosuria for some years while taking a normal diet. ${ }^{1}$ In the twentieth century and particularly since the introduction of insulin there have been numerous case reports of remissions in diabetes. Most commonly these are to be seen in children, ${ }^{2}$ but they are not unusual after the initial treatment of adult, insulin-requiring patients. ${ }^{3}$ When patients present with the severer metabolic disturbances they are treated with insulin, and the dose of insulin required in the initial stage of therapy can frequently be reduced considerably over the succeeding weeks. In some patients insulin can be withdrawn without any increase in blood sugar levels, though it is a bold physician who does not continue with some form of therapy, whether with oral hypoglycaemic agents or simply restriction of carbohydrate.

This state of remission lasts for a variable time, rarely for several years, and even more rarely for such a long time that the term recovery becomes appropriate. ${ }^{4} \mathrm{~A}$ most dramatic example is the case described by J. B. Field and his colleagues ${ }^{5}$ of a 15-year-old girl who developed diabetes and subsequently severe insulin resistance. For a time she received up to 38,000 units of insulin daily, yet she eventually had a gradual but complete remission of both her insulin resistance and her diabetes. Whether therapy can prolong the period of remission is undecided, for the duration is so variable, and no controlled trial has been performed. However, there has been experimental work to support the maintenance of carbohydrate restriction during the remission. R. E. Haist and his colleagues ${ }^{6}$ concluded that fasting, a fat diet, or insulin protected animals against diabetes after partial pancreatectomy or anterior pituitary injections. These experiments have also been cited to justify vigorous treatment of the diabetes initially. ${ }^{\text {? }}$

More recently studies have been made on insulin levels in the blood in relation to clinical remission, including the response to glucose, tolbutamide, and glucagon. ${ }^{8-10}$ Of the patients studied, one had been treated with oral hypoglycaemic agents, except for a short period on insulin, ${ }^{8}$ and the other five with insulin. The results in each of the investigations were similar: insulin secretion was greater during periods of remission, though still modest in comparison with normal subjects. In the patients treated with insulin, glucose tolerance improved sufficiently to allow withdrawal of insulin, but it never became entirely normal. While "juvenile-onset" diabetes is now generally regarded as associated with absolute or relative deficiency of insulin these studies in remission suggest that, at least in some patients, there is sufficient functional activity in the pancreas to cope with an unstressed situation. This should encourage those who are looking for some means of delaying or even preventing the hitherto inexorable progression of the disorder. The studies also suggest that the intermittent diabetes may result from a temporary reduction in insulin production. How this may come about is a matter for speculation, though some suspicion may reasonably be cast upon the adrenal medullary hormones, which can undoubtedly inhibit insulin secretion. ${ }^{11}$ Current studies of the metabolism of the islets of Langerhans may eventually throw some light on the mechanisms concerned.

Remissions in so-called maturity onset diabetes are much more difficult to identify, partly because maturity onset diabetes itself defies adequate definition. ${ }^{12}$ L. H. Newburgh and J. W. Conn ${ }^{13}$ reported the return to normal of glucose

1 Allan, F. N., Fournal of the American Dietetic Association, 1930, 6, 1.

Brush, J. M., American fournal of Diseases of Children, 1944, 67, 429.

3 Lukens, F. D. W., and Dohan, F. C., Pennsylvania Medical fournal, 1944,

48, 24. Lister, J., Lancet, 1966, 1, 386.

5 Field, J. B., Johnson, P., and Herring, B., fournal of Clinical Investigation, $1961,40,1672$.

- Haist, R. E., Campbell, J., and Best, C. H., New England fournal of Medicine, 1940, 223, 607 .

' Jackson, R. L., Boyd, J. D., and Smith, T. E., American fournal of Diseases of Children, 1940, $\mathbf{5 9}, 332$.

- Kosaka, K., Hagura, R., Saito, R., Tsukamoto, F., and Kuzuya, T., Diabetes, 1969, 18, 487.

- Johansen, K., and Ørskov, H., British Medical fournal, 1969, 1, 676.

10 Genuth, S., Diabetes, 1970, 19, 116.

11 Porte, D., Graber, A. L., Takeshi, K., and Williams, R. H., fournal of Clinical Investigation, 1966, 45, 228.

12 Keen, H., and Jarrett, R. J., in The New Management of Stable Adult Diabetes, Ed. C. Weller, p. 3. Springfield, Ill., Thomas, 1969.

13 Newburgh, L. H., and Conn, J. W., fournal of the American Medical Association, 1939, 112, 7.

14 O'Sullivan, J. B., and Hurwitz, D., Archives of Internal Medicine, 1966, 117, 769 . 
tolerance of obese diabetics who achieved adequate loss of weight; such treatment-induced remission is familiar to most doctors. But to bedevil the assessment of therapy in diabetes it is now clear that spontaneous remission may occur not uncommonly. J. B. O'Sullivan and D. Hurwitz ${ }^{14}$ studied 83 women found to have glucose tolerance tests diagnostic of diabetes (by the stringent criteria of the U.S. Public Health Service) who were followed for several years without specific treatment, though they were advised of the benefits of normal weight. Spontaneous remissions to normal glucose tolerance were frequent, both in obese and non-obese subjects, and two-thirds of the patients with remissions had had no change in weight or had actually gained weight. So it seemed unlikely that dietary restriction could be invoked as responsible for the remissions. O'Sullivan and Hurwitz thought it probable that the patients they were observing did have abnormal glucose tolerance (or diabetes) and not fluctuations within the normal range and that use of the term remission was appropriate. The physiology of the remission is unexplained. The clinical lesson, though, is clear-that the assessment of the effects of treatment in diabetes of this nature requires rigorous controls.

\section{The Heart in London}

This coming week the Sixth World Congress of Cardiology is to be held at the Festival Hall in London under the patronage of the Queen. Five and a half days of discussions and lectures, many going on simultaneously, will give a thorough conspectus of research into the cardiovascular diseases. Though the range and complexity of the subjects to be discussed are remarkable, the fact remains that this group of diseases is the commonest cause of death in all the developed countries of the world. It is fast becoming so in other countries as they conquer those old infectious scourges of mankind: tuberculosis, malaria, smallpox, and cholera. The conquest of the heart's ailments is only beginning.

The British Cardiac Society and the British Heart Foundation are the hosts and organizers of the congress. Its president is Sir John McMichael, whose pioneering work at the Royal Postgraduate Medical School in London until he retired in 1966 attracted to that institution visitors and research students from all over the world. Distinguished cardiologists form the organizing committee-namely, Professor J. Shillingford (chairman), Professor J. F. Goodwin (deputy chairman), Professor C. T. Dollery (chairman of scientific programme committee), and Dr. W. Somerville (chairman of social events committee).

The sponsor of the congress is the International Society of Cardiology, a body initiated in 1946 at a meeting of cardiologists held in Mexico City. It now has over 60 national societies and promotes a world congress every four years in different capital cities. Thus as well as giving cardiologists an excuse to see the sights of the world these meetings bring them to countries where at least some of the cardiological problems will be unfamiliar or receive a different emphasis from what they are accustomed to in their homeland.

Great Britain itself has long enjoyed a reputation for vitality in cardiovascular research and for a sense of proportion in the management of patients. Evidence of lively research is to be found in the papers appearing in the British Heart fournal, one of the specialist journals published in association with the B.M.F., and now also in Cardiovascular Research, started in
1967 to accommodate the more technical papers in basic research. These journals are to publish abstracts of some of the congress papers.

At the congress, as in published papers, the emphasis is naturally enough on haemodynamics, electronics, and biochemistry. If the British contributions to this congress succeed also in communicating a hint of the care taken in this country to manage patients as individuals they will have imparted something worthwhile from a trusted tradition.

\section{Control of Waste}

Paradoxically, the present wave of public concern about environmental pollution comes at the end of a decade of real improvement in the quality of the atmosphere in Britain. Visible air pollution has been greatly reduced by the introduction of smokeless zones, while sulphur dioxide concentrations at ground level have fallen by an average of $40 \%$ in the last ten years. Adverse comments ${ }^{1}$ by the Chief Inspector in the Annual Report on Alkali \&c. Works on "exaggerated claims" and "gloomy prophecies" made by conservationists have been widely reported. His reaction is understandable in view of the success of his inspectorate in controlling industrial waste.

Nevertheless, the report correctly states that "the problems of air pollution control are mainly economic." Only if there is enough pressure of public opinion will standards of industrial pollution be raised and will adequate means be found for the innocuous disposal of plastic refuse, old motor tires, and the other garbage of an affluent society. Doctors have a particular interest in clean air because of its importance in preventing the "English disease," chronic bronchitis, yet some local authorities still resist smokeless zones. ${ }^{2}$

Those responsible for controlling pollution may find the present fashionable interest inconvenient and time consuming. But in the long term increased public awareness can lead only to better standards of cleanliness in our environment.

\section{Bath Meeting}

The Annual Clinical Meeting of the B.M.A. at Bath next month has been arranged in conjunction with the Royal College of Physicians of London and the Heberden Society. Rheumatic disorders are historically associated with Bath, and a substantial part of the programme, printed this week in the Supplement, is made up of sessions on polymyalgia rheumatica, complications of rheumatoid disease, and total hip replacement. More general subjects include coronary care, abdominal pain in childhood, and a session on "Drugs for the Individual," and informal discussion sessions have been arranged on common problems such as the ischaemic leg and hypertension in pregnancy. The opening address on space medicine is to be given by Wing-Commander D. I. Fryer, of the R.A.F. Institute of Aviation Medicine.

Bath's eighteenth century elegance has been preserved nearly intact, and among the attractions of the surrounding country are Glastonbury and the lions of Longleat. With its 2,000-year association with medicine a better setting than Bath for a Clinical Meeting is hard to imagine.

\footnotetext{
1 Ministry of Housing and Local Government, Scottish Development Department, Welsh Office. 106th Annual Report on Alkali E'c. Works, 1969. London, H.M.S.O., 1970.
} 